\title{
ON THE PRINCIPLE OF OPTIMALITY FOR LINEAR STOCHASTIC DYNAMIC SYSTEM
}

\author{
Yakup H. HACI, Muhammet CANDAN and Aykut OR \\ Department of Mathematics, Canakkale Onsekiz Mart University, Canakkale, Turkey
}

\begin{abstract}
In this work, processes represented by linear stochastic dynamic system are investigated and by considering optimal control problem, principle of optimality is proven. Also, for existence of optimal control and corresponding optimal trajectory, proofs of theorems of necessity and sufficiency condition are attained.
\end{abstract}

\section{KEYWORDS}

Optimal control problem, linear stochastic dynamic systems, optimal trajectory.

\section{INTRODUCTION}

Linear stochastic dynamic system (LSDS) is the one of the most developing field of discrete system theory. Since LSDS theory has seen an important growth due to its interaction with fields like base of computer science, graph theory, coding theoy, diagnosing problem in the discrete events, imitation problem etc. Thus, it requires more detailed investigation.

The mathematical theory of optimal control consisting all the continuous and discrete systems is strong studied more than 40 years. L. S. Pontryagin et al. first obtained basic results of the theory of optimal control and then this theory was developed and simplificated by other academicians [1-4]. Gaishun presented a detailed works regarding the optimal control problem to multidimensional differential equation[5]. Gabasov et al. considered a linear quadratic optimal control problem with geometric constraints on the control actions and gave some novel numerical methods for both optimal program and positional solutions[6]. Also, they studied a problem of linear optimal control under uncertainty[7]. We firstly investigated the optimal discrete processes given by nonlinear multiparameter stochastic dynamic systems.[8-9]

The present article is devoted the determination of optimal control and corresponding trajectory with necessary and sufficient condition theorems in the linear stochastic dynamic system. First section introduces general definition and basic concepts of the LSDS. Then in the second section,existence of optimal control and corresponding trajectory is discussed as necessary and sufficient conditions. Finally, main idea and aim of this paper is presented.

\section{LINEAR STOCHASTIC DYNAMIC SYSTEM}

LSDS is generalization of multi-parametric finite sequential dynamic system but it contains a probability variable. General form of this system is defined by[5]:

$$
K=<X, S, Y, s^{0}, p(\omega), F_{v}(\cdot)>\quad v=1,2, \ldots k
$$


where $X=[G F(2)]^{r}, S=[G F(2)]^{m}$ and $Y=[G F(2)]^{q}$ are input, state and output index (alphabet) respectively; $s^{0}$ is initial state vector, $p(\omega)$ is determitistic discrete probability distribution. $\left(\Omega=\left\{\omega_{1}, \omega_{2}, \ldots, \omega_{p}\right\}\right.$ is finite set, $\left.\quad p(\omega)=\left\{p\left(\omega_{i}\right): \omega_{i} \in \Omega, \sum_{\omega_{i} \in \Omega} p\left(\omega_{i}\right)=1\right\}\right)$ Characteristic Boolean vector function [12] denoted by $F_{v}(\cdot)=\left\{F_{v_{1}}(\cdot), \ldots, F_{v_{m}}(\cdot)\right\}$ which is also known as transfer functions is equal;

$$
F_{v}(c, s(c), x(c) \oplus \omega(c))=\xi_{v} s(c)=\Phi_{v}(c) s(c) \oplus \Psi_{v}(c)(x(c) \oplus \omega(c)) \quad \mathrm{v}=1, \ldots, \mathrm{k}
$$

where the points $c=\left(c_{1}, c_{2}, \ldots, c_{k}\right) \in G_{d}=\left\{c \mid c \in Z^{k}, c_{1}^{0} \leq c_{1} \leq c_{1}^{L_{1}}, \ldots, c_{k}^{0} \leq c_{k} \leq c_{k}^{L_{k}}, c_{i} \in Z\right\}$

are points in $Z^{k}$, determining position $L_{i},(i=1,2, \ldots k$ positive integer $)$ is the duration of the stage $i$ of this process. $Z$ is set of integers, for $s(c) \in S, x(c) \in X ; s(c), x(c)$ are defined over the set $G_{d}$ as an $m$ and $r$ dimensional state and input vectors. $\xi_{v} s(c)$ is a shift operator[5],[11] defined as:

$\xi_{v} s(c)=s\left(c+e_{v}\right), e_{v}=(0, \ldots, 0, \stackrel{v}{1}, 0, \ldots, 0), \mathrm{v}=1, \ldots, \mathrm{k}$

$\left\{\Phi_{v}(c), v=1, \ldots k\right\},\left\{\Psi_{v}, v=1, \ldots, k\right\}$ are characteristic Boolean transition matrices of size $m \times m$ and $m \times r$ respectively. $\omega$ is a random variable. $G F(2)$ is Galois field and all operations are on $G F(2)$ in this study.

In LSDS, each random variable $\omega$ has a special case. In (2) $\omega$ is an input variable . However, $\omega$ can be a set of all possible states in the synthesis of dynamic systems.

Moreover, the state of the system depends on the random variable $\omega$ which affects not only parameters of the LSDS but also the input variables.

The discrete optimal processes given by LSDS are characterized by functional:

$$
J(x)=M_{\omega}\left\{a s^{T}\left(c^{L}\right)\right\}
$$

Where $M_{\omega}\{$.$\} is a mathematical expected value of \omega, a=\left(a_{1}, a_{2}, \ldots, a_{m}\right)$ is given Boolean vector and $s^{T}\left(c^{L}\right)$ is transpose of $s\left(c^{L}\right)$. It is clearly seen that for $x(c) \in X$, the number of equations in (1) is more than the number of unknowns. Therefore, the considered problem can be determined as an admissible optimal control problem [4], [6-7].

Definition. If there is a unique solution in (2) for $x(c) \in X$, then $x(c)$ is called an admissible control[8-9].

Let $\hat{X}$ be the set of admissible administrator functions defined from $\hat{G}_{d}=G_{d} \backslash\left\{c^{L}\right\}$ into $[G F(2)]^{r}$

\section{Optimal Control Problem And Principle Of Optimality}

For linear stochastic dynamic system, we can analyze the following problem: 
For a given linear stochastic sequential dynamic system, to move from an initial state $s^{0}$ to a desired terminal state $s^{*}\left(c^{L}\right)$ in L steps, a control $x(c) \in \hat{X}$ must exist such that the functional (4) has a minimum value. In other words;

$$
\begin{aligned}
& \xi_{v} s(c)=\Phi_{v}(c) s(c) \oplus \Psi_{v}(c)(x(c) \oplus \omega(c)) \quad \mathrm{v}=1, \ldots, \mathrm{k} \\
& s\left(c^{0}\right)=s^{0} \\
& x(c) \in \hat{X}, \quad c \in \hat{G}_{d} \\
& J(x)=M_{\omega}\left\{a s^{T}\left(c^{L}\right)\right\} \rightarrow \min
\end{aligned}
$$

Assume that condition of the unique solution [5],[8] in (2) is holds. Then sequential dynamic system can written as below;

$\varphi(c)=\Phi_{v}(c) \varphi\left(\xi_{v} c\right), c \in G_{d}, \mathrm{v}=1, \ldots, \mathrm{k}$,

and by considering this system, if Boolean function is constructed as follows,

$$
h_{v}\left(c, x(c) \oplus \omega(c), \varphi\left(\xi_{v} c\right)\right)=\varphi\left(\xi_{v} c\right) \Psi_{v}(c)(x(c) \oplus \omega(c)), \mathrm{v}=1, \ldots, \mathrm{k},
$$

Then by using this function we form the following sum:

$$
\hat{h}=\sum_{L\left(c^{l}, c^{L}\right)} \sum_{v=1}^{k} h_{v}\left(c, x(c) \oplus \omega(c), \varphi\left(\xi_{v} c\right)\right) \Delta_{2} c_{v}
$$

Where $\Delta_{2}$ is the first Boolean difference

Theorem I Suppose that the system in (2) has an exact solution then the sum in (7) is path independent.

Proof It is precise that if the equation

$$
\begin{aligned}
& \varphi\left(\xi_{v} c\right) \Psi_{v}(c)(x(c) \oplus \omega(c)) \oplus \varphi\left(\xi_{v^{\prime}} \xi_{v} c\right) \Psi_{v^{\prime}}\left(\xi_{v} c\right)\left(x\left(\xi_{v} c\right) \oplus \omega\left(\xi_{v} c\right)\right)= \\
& \left.\left.=\varphi\left(\xi_{v^{\prime}} c\right) \Psi_{v^{\prime}}(c)(x(c) \oplus \omega(c)) \oplus \varphi\left(\xi_{v} \xi_{v^{\prime}} c\right) \Psi_{v}\left(\xi_{v^{\prime}} c\right)\left(x\left(\xi_{v^{\prime}} c\right)\right) \oplus \omega\left(\xi_{v^{\prime}} c\right)\right)\right) \quad v, v^{\prime}=1,2, \ldots, k
\end{aligned}
$$

holds, then Boolean sum in (7) is path independent. By using equivalent equations like:

$$
\varphi(c)=\Phi_{v}(c) \varphi\left(\xi_{v} c\right), c \in G_{d},
$$

It is found[4]

$$
\begin{gathered}
\varphi\left(\xi_{v^{\prime}} \xi_{v} c\right)=\left(\Phi_{v^{\prime}}\left(\xi_{v} c\right)\right)^{-1}\left(\Phi_{v}(c)\right)^{-1} \varphi(c), \\
\varphi\left(\xi_{v} \xi_{v^{\prime}} c\right)=\left(\Phi_{v}\left(\xi_{v^{\prime}} c\right)\right)^{-1}\left(\Phi_{v^{\prime}}(c)\right)^{-1} \varphi(c), v, v^{\prime}=1,2, \ldots, k .
\end{gathered}
$$

By substituting (10) into (8),

$$
\begin{aligned}
& \left(\Phi_{v}(c)\right)^{-1} \Psi_{v}(c)(x(c) \oplus \omega(c)) \varphi(c) \oplus\left(\Phi_{v^{\prime}}\left(\xi_{v} c\right)\right)^{-1}\left(\Phi_{v}(c)\right)^{-1} \Psi_{v^{\prime}}\left(\xi_{v} c\right)\left(x\left(\xi_{v} c\right) \oplus \omega\left(\xi_{v} c\right)\right) \varphi(c) \\
& =\left(\Phi_{v^{\prime}}(c)\right)^{-1} \Psi_{v^{\prime}}(c)(x(c) \oplus \omega(c)) \varphi(c) \oplus \\
& \left(\Phi_{v}\left(\xi_{v^{\prime}} c\right)\right)^{-1}\left(\Phi_{v^{\prime}}(c)\right)^{-1} \Psi_{v^{\prime}}\left(\xi_{v^{\prime}} c\right)\left(x\left(\xi_{v^{\prime}} c\right) \oplus \omega\left(\xi_{v^{\prime}} c\right)\right) \varphi(c) .
\end{aligned}
$$


International Journal in Foundations of Computer Science \& Technology (IJFCST) Vol.6, No.1, January 2016

Multiplying the above equations from the both sides by

$$
\Phi_{v}\left(\xi_{v^{\prime}} c\right) \Phi_{v^{\prime}}(c)=\Phi_{v^{\prime}}\left(\xi_{v} c\right) \Phi_{v}(c)
$$

(11) forms,

$\Phi_{v^{\prime}}\left(\xi_{v} c\right) \Psi_{v}(c)(x(c) \oplus \omega(c)) \varphi(c) \oplus \Psi_{v^{\prime}}\left(\xi_{v} c\right)\left(x\left(\xi_{v} c\right) \oplus \omega\left(\xi_{v} c\right)\right) \varphi(c)$

$=\left(\Phi_{v}\left(\xi_{v^{\prime}} c\right) \Psi_{v^{\prime}}(c)(x(c) \oplus \omega(c)) \varphi(c) \oplus \Psi_{v}\left(\xi_{v^{\prime}} c\right)\left(x\left(\xi_{v^{\prime}} c\right)\right) \oplus \omega\left(\xi_{v^{\prime}} c\right)\right) \varphi(c)$

Now the equation (13) holds for every $\varphi(c)$, it is written by,

$$
\begin{aligned}
& \Phi_{v^{\prime}}\left(\xi_{v} c\right) \Psi_{v}(c)(x(c) \oplus \omega(c)) \oplus \Psi_{v^{\prime}}\left(\xi_{v} c\right)\left(x\left(\xi_{v} c\right) \oplus \omega\left(\xi_{v} c\right)\right)= \\
= & \Phi_{v}\left(\xi_{v^{\prime}} c\right) \Psi_{v^{\prime}}(c)(x(c) \oplus \omega(c)) \oplus \Psi_{v^{\prime}}\left(\xi_{v^{\prime}} c\right)\left(x\left(\xi_{v^{\prime}} c\right) \oplus \omega\left(\xi_{v^{\prime}} c\right)\right)
\end{aligned}
$$

The equation (14) is the exact solution of the system in (2). Since the solution is the exact, the equation (8) holds and the Boolean sum in (7) is path independent.

Let $\hat{L}\left(c^{0}, c^{1}, \ldots, c^{L}\right)$ be a piecewise curve connecting the point $c^{0}$ to the point $c^{L}$. In the $l^{\text {th }}$ step where $0 \leq l<L$, assigning a value $v_{t}$ to the piece of curve from $c^{l}$ to the point $c^{l+1}$. Then the Boolean sum is in (7) has the following form:

$$
\hat{h}=\sum_{\left(c^{l}, c^{L}\right)} h_{v_{t}}\left(c^{t-1}, x\left(c^{t-1}\right) \oplus \omega\left(c^{t-1}\right), \varphi\left(c^{t}\right)\right)=\sum_{t=l+1}^{L} h_{v_{t}}\left(c^{t-1}, x\left(c^{t-1}\right) \oplus \omega\left(c^{t-1}\right), \varphi\left(c^{t}\right)\right)
$$

Hamilton- Pontryagin functional

$$
H(\varphi(c), s(c))=\varphi(c) s(c), \quad c \in G_{d}
$$

can be involved, which is similar to Boolean functional.

Theorem II A control $x^{0}\left(c^{l}, c^{L-1}\right)=\left\{x^{0}\left(c^{l}\right), x^{0}\left(c^{l+1}\right), \ldots, x^{0}\left(c^{L-1}\right)\right\}$ and corresponding trajectory $s^{0}\left(c^{l}, c^{L}\right)=\left\{s^{0}\left(c^{l}\right), s^{0}\left(c^{l+1}\right), \ldots, s^{0}\left(c^{L}\right)\right\}$ are optimal if and only if,

$$
H\left(\varphi^{0}\left(c^{l}\right), s^{0}\left(c^{l}\right)\right)=\sum_{t=l+1}^{L} h_{v_{t}}\left(c^{t-1}, \varphi^{0}\left(c^{t}\right), x^{0}\left(c^{t-1}\right) \oplus \omega\left(c^{t-1}\right)\right)
$$

Where $\varphi^{0}\left(c^{t}\right), t=l, l+1, \ldots, L-1$ is obtained from the equation below;

$$
\varphi(c)=\Phi_{v}(c) \varphi\left(\xi_{v} c\right), c \in G_{d}, v=1,2, \ldots ., k
$$

With the aid of equation $\varphi^{0}\left(c^{L}\right)=a$.

Proof Firstly, if necessity condition is proven then, from (7) for $l+1 \leq t<L$,

$$
\begin{aligned}
H\left(\varphi^{0}\left(c^{t}\right), s^{0}\left(c^{t}\right)\right) & =\varphi^{0}\left(c^{t}\right) s^{0}\left(c^{t}\right) \\
& =\varphi^{0}\left(c^{t}\right) \Phi_{v_{t}}\left(c^{t-1}\right) s^{0}\left(c^{t-1}\right) \oplus \varphi^{0}\left(c^{t}\right) \Psi_{v_{t}}\left(c^{t-1}\right)\left(x^{0}\left(c^{t-1}\right) \oplus \omega\left(c^{t-1}\right)\right) \\
& =\varphi^{0}\left(c^{t-l}\right) s^{0}\left(c^{t-l}\right) \oplus \varphi^{0}\left(c^{t}\right) \Psi_{v_{t}}\left(c^{t-1}\right)\left(x^{0}\left(c^{t-1}\right) \oplus \omega\left(c^{t-1}\right)\right)
\end{aligned}
$$


International Journal in Foundations of Computer Science \& Technology (IJFCST) Vol.6, No.1, January 2016

$$
=H\left(\varphi^{0}\left(c^{t-l}\right), s^{0}\left(c^{t-l}\right)\right) \oplus h_{v_{t}}\left(c^{t-1}, x^{0}\left(c^{t-1}\right) \oplus \omega\left(c^{t-1}\right), \varphi^{0}\left(c^{t}\right)\right) .
$$

When the sum is taken from $l+1$ to $L$, the following equalities are;

$$
\sum_{t=l+1}^{L} H\left(\varphi^{0}\left(c^{t}\right), s^{0}\left(c^{t}\right)\right)=\sum_{t=l+1}^{L} H\left(\varphi^{0}\left(c^{t-1}\right), s^{0}\left(c^{t-1}\right)\right) \oplus \sum_{t=l+1}^{L} h_{v_{t}}\left(c^{t-1}, x^{0}\left(c^{t-1}\right) \oplus \omega\left(c^{t-1}\right), \varphi^{0}\left(c^{t}\right)\right)
$$

and

$$
H\left(\varphi^{0}\left(c^{L}\right), s^{0}\left(c^{L}\right)\right)=H\left(\varphi^{0}\left(c^{l}\right), s^{0}\left(c^{l}\right)\right) \oplus \sum_{t=l+1}^{L} h_{v_{t}}\left(c^{t-1}, x^{0}\left(c^{t-1}\right) \oplus \omega\left(c^{t-1}\right), \varphi^{0}\left(c^{t}\right)\right)
$$

are obtained.

On the contrary,

$$
\Phi\left(s^{0}\left(c^{L}\right)\right)=a s^{0}\left(c^{L}\right)=\varphi^{0}\left(c^{L}\right) s^{0}\left(c^{L}\right)=H\left(\varphi^{0}\left(c^{L}\right), s^{0}\left(c^{L}\right)\right)
$$

Since $s^{0}\left(c^{L}\right)$ is an optimal state,

$$
H\left(\varphi^{0}\left(c^{L}\right), s^{0}\left(c^{L}\right)\right)=0
$$

is found.

Therefore, (17) is valid. This completes the proof of the necessity condition.

Sufficiency: The sufficient condition is proven in a similar form as of necessity and from (21) the desired result is obtained.

Under assumption of equation (2) in equilibrium state, the following theorem can be given;

Theorem III A control $x^{0}(c)$ and corresponding trajectory $s^{0}(c)$ are optimal in (1) if and only if,

$$
\sum_{\left(c^{0}, c^{L}\right)} \sum_{v=1}^{k} h_{v}\left(c, x^{0}(c) \oplus \omega^{0}(c), \varphi^{0}\left(\xi_{v} c\right)\right) \Delta_{2} c_{v} \times \overline{\sum_{\left(c^{0}, c^{L}\right)} \sum_{v=1}^{k} h_{v}\left(c, x(c) \oplus \omega(c), \varphi^{0}\left(\xi_{V} c\right)\right) \Delta_{2} c_{v}=0}
$$

Where the bar denotes the complement of this sum applied on the piecewise curve connecting $c^{0}$ to $c^{L}$ with all admissible controls.

Proof Necessity: By the exact solution of (2),

$$
\begin{aligned}
& s\left(c^{L}\right)=\sum_{\left(c^{0}, c^{L}\right)} \sum_{v=1}^{K} \hat{\Phi}\left(c^{L}, \xi_{v} c\right) \Psi_{v}(c)(x(c) \oplus \omega(c)) \Delta_{2} c_{v} \\
& J(x)=a s\left(c^{L}\right)=\sum_{\left(c^{0}, c^{L}\right)} \sum_{v=1}^{K} a \hat{\Phi}\left(c^{L}, \xi_{v} c\right) \Psi_{v}(c)(x(c) \oplus \omega(c)) \Delta_{2} c_{v}
\end{aligned}
$$

Let $\varphi\left(\xi_{v} c\right)=a \hat{\Phi}\left(c^{L}, \xi_{V} c\right)$ where $c=c^{0}, c^{1}, \ldots ., c^{L-1}$ and $\hat{\Phi}\left(c^{L}, \xi_{V} c\right)=S\left(c^{L}\right) S^{-1}\left(\xi_{v} c\right)$. 
$S(c)$ is the fundemental matrix operator [5] of the system

$$
\begin{aligned}
& \xi_{v} s(c)=\Phi_{v}(c) s(c), \\
& s\left(c^{0}\right)=s^{0}
\end{aligned}
$$

at the point $c$. Thus $\varphi^{0}\left(c^{L}\right)=a$ and for the optimal control, minimum is taken as;

$$
\sum_{\left(c^{0}, c^{L}\right)} \sum_{v=1}^{k} h_{v}\left(c, x^{0}(c) \oplus \omega^{0}(c), \varphi^{0}\left(\xi_{v} c\right)\right) \Delta_{2} c_{v}=\min \sum_{\left(c^{0}, c^{L}\right)} \sum_{v=1}^{k} h_{v}\left(c, x(c) \oplus \omega(c), \varphi^{0}\left(\xi_{v} c\right)\right) \Delta_{2} c_{v}
$$

Considering the curve connecting $c^{0}$ to $c^{L}$ over the all admissible controls $x(c),(16)$ equals to Boolean equality,

$$
\sum_{\left(c^{0}, c^{L}\right)} \sum_{v=1}^{k} h_{v}\left(c, x(c) \oplus \omega(c), \varphi^{0}\left(\xi_{v} c\right)\right) \Delta_{2} c_{v} \geq \sum_{\left(c^{0}, c^{L}\right)} \sum_{v=1}^{k} h_{v}\left(c, x^{0}(c) \oplus \omega^{0}(c), \varphi^{0}\left(\xi_{v} c\right)\right) \Delta_{2} c_{v}
$$

Alternatively, the inequality (29) equals to;

$$
\overline{\sum_{\left(c^{0}, c^{L}\right)} \sum_{v=1}^{k} h_{v}\left(c, x(c) \oplus \omega(c), \varphi^{0}\left(\xi_{v} c\right)\right) \Delta_{2} c_{v}} \sum_{\left(c^{0}, c^{L}\right)} \sum_{v=1}^{k} h_{v}\left(c, x^{0}(c) \oplus \omega^{0}(c), \varphi^{0}\left(\xi_{v} c\right)\right) \Delta_{2} c_{v}=0
$$

Sufficiency: Assuming (30), if substituting $\varphi\left(\xi_{v} c\right)=a \hat{\Phi}\left(c^{L}, \xi_{v} c\right)$ into (29),

$$
\sum_{\left(c^{0}, c^{L}\right)} \sum_{v=1}^{K} a \hat{\Phi}\left(c^{L}, \xi_{v} c\right) \Psi_{v}(c)\left(x^{0}(c) \oplus \omega^{0}(c)\right) \Delta_{2} c_{v} \leq \sum_{\left(c^{0}, c^{L}\right)} \sum_{v=1}^{K} a \hat{\Phi}\left(c^{L}, \xi_{v} c\right) \Psi_{v}(c)(x(c) \oplus \omega(c)) \Delta_{2} c_{v}
$$

it is clear that $x(c)$ is an arbitrary admissible control and from $(31) x^{0}(c)$ is determined as the optimal control.

\section{Conclusions}

It is shown that optimal control problem for processes with multi parametric linear stochastic dynamical systems are studied. It is evident that there is an absolutely a control and corresponding such that the considered system has a minimum value while connecting the initial state with terminal state. Furthermore, theorems involving necessary and sufficient conditions for optimality are proven. Thus, the results obtained in this paper will be contribute to application.

\section{REFERENCES}

[1] Pontryagin, L. S., Boltyanskii, V. G., Gamkrelidze, Mishchenko., (1962) The Mathematical Theory of Optimal Processes, Interscience Publishers, New York,

[2] Farajov, R. G., (1975) Linear Sequential Machines, Sov. Radio, (Russian).

[3] Gill, A., (1975) Linear Sequential Machines, Nauka, (Russian)

[4] Boltyanskii, V. G., (1978) Optimal Control of Discrete Systems, John Willey, New York, p.363.

[5] Gaishun, I. V., (1983) Completely Solvable Multidimensional Differential Equations, Nauka and Tekhnika, Minsk, p.231. 
[6] Gabasov,R., Kirillova, F. M., Paulianok, N. S. "Optimal Control of Linear Systems on Quadratic Performance Index', Appl. and Comput. Math., V.7, N.1, 2008, pp.4-20.

[7] Gabasov,R., Kirillova, F. M., Payasok, E. S. 'Robust Optimal Control on Imperct Measurements of Dynamic Systems States', Appl. and Comput. Math., V.8, N.1, 2009, pp.54-69.

[8] Hac1, Y., Ozen, K., (2009) "Terminal Control Problem for Processes Represented by Nonlinear Multiparameter Binary Dynamic System”, Control and Cybernetics, Vol. 38, No. 3, pp625-633.

[9] Hacı Y., Candan M.,(2014) Optimal Control Problem for Processes Represented by Stochastic Sequential Machine." Internatinal Journal on Cybernetics and Informatics. Vol 3 no 4 pp. 21-26

[10] Yermolyev, Y. M., (1976) Stochastic Programming Methods, Nauka (in Russian), p.240.

[11] Burden, R. L., Dauglas, J. Numerical Analysis, PWS Publishing Company, Boston, 1985.

[12] Yablonsky, S. V., (1989) Introduction to Discrete Mathematics, Mir Publishers, Moscow, p.9.

[13] Bellman, R., (1957) Dynamic Programming, Princeton University Press, Princeton, p.12

[14] Anderson, J. A., (2004) Discrete Mathematics with Combinatorics, Prentice Hall, New Jersey, p.45

\section{AUTHOR}

Yakup H. HACI ${ }^{1}$ received Ph.D at physics and mathematics in Azerbaijan NationalAcademy of Sciences at the Institute of Cybernetics. He is currently working as professor at the department of Mathematics, in Canakkale Onsekiz Mart University. His primary research interests are in the areas of the theory of multi parametric binary sequential machines, linear and nonlinear optimization and discrete mathematics. He is an author(co-author) of over 38 papers.

Muhammet CANDAN ${ }^{2}$ received his M.Sc degree from Canakkale Onsekiz Mart University, Canakkale, Turkey, in 2012. He is currently Ph.D. candidate and working as a research assistant in Canakkale Onsekiz Mart University. His research interests cover applied mathematics, finite dynamical systems, graph theory.

Aykut $\mathbf{O R}^{3}$ received his Ph.D at mathematics from Canakkale Onsekiz Mart University, Turkey, in 2014. $\mathrm{He}$ is currently working as a research assistant in Canakkale Onsekiz Mart University. His mainly research interests cover applied game theory, interval matrix, bimatix games. 